\title{
THE DESIGN OF FRUIT AUTOMATED SORTING SYSTEM
}

\author{
Pingju Ge ${ }^{*}$, Qiulan Wu, Yongxiang Sun \\ College of information Science \& Engineering, Shandong agricultural university, Taian \\ 271018, China \\ * Corresponding author, Address: College of information Science \& Engineering, Shandong \\ agricultural university, Taian 271018, Shandong, P. R. China, Tel: +86-538-8242497, \\ Email: gepj2@163.com
}

Abstract: The article introduced the flow and design scheme of fruit automated sorting system, the computer technology and automatic technology is used in agriculture production and management. In this system, the application of some technology, such as the detection and control technology, the digital image processing technology, the computer peripheral device and interface technology, Pattern Recognition technology etc, is used in the fruit automated sorting system.

Keywords: the fruit, automated sorting system, automation, the digital image processing, Pattern Recognition

\section{INTRODUCTION}

With the development of modern agriculture \& forestry and the enhance of industrial standard, the output of fruits from inner country and abroad are being raised swiftly. On the one hand, the fruits from different district are sold in local area, on the other hand, more plenty of fruits are sold to other district even abroad, and these fruits demand being packed according to different quality. In order to package these fruits, we must to design a fruit automated sorting system, because if these fruits were packed by hand, one the one hand, The task is hard and it is inefficient; on the other hand, it is not meet the demands of modern fruit package, which is need to be Multi-layer or high standard or high sanitary standard. 
To advanced country after second war, the automated sorting system is one of the essential equipment of Logistics centre, Distribution Centre, or circulation centre (Huang, 2002), it was used in American and Europe in the 1960s, automated sorting system was used in Japan in 1970s (Xu, 2002). At the present, automated sorting system is also success used in post office besides above field. With the improvement of modernization standard, it is widely used in more and more field. at the present, almost no success using about fruit automated sorting system because it concern a lots of knowledge and subject, at the same time, it demand more higher level of artificial. At present, we can only see the news about Fruitonics company, which have designed a set of fruit automated sorting system.

\section{THE HARDWARE DESIGN OF FRUIT AUTOMATED SORTING SYSTEM}

In the course of designing of the System, all kinds of factor are comprehensively thought when fruits are sorted. All kinds of technology, such as detection and control technology, the computer peripheral equipment and interface technology, the digital image processing technology and Pattern Recognition technology, is used, at the same time, advanced algorithm is used in the course of pattern identification, and it improve the accurate rate of fruit sorting.

\subsection{The hardware consists of fruit automated sorting system}

There are all sorts of automated sorting system, and its standard is different. In general, an automated sorting system is consists of seven partition $(\mathrm{Xu}, 2002)$, it is: collection Conveyor, feed Conveyor, the Setting Device Of sorting instruction, confluence device, sorting conveyer, sorting and discharge cargo port, computer controller (Fig. 1).

\subsection{The consists of hardware and work principles of fruit automated sorting system}

The fruit automated sorting system is based of the traditional automated sorting system, but it is different of the traditional automated sorting system, it is an automated sorting system which is high intelligent, real-time, Multilayer, multi knowledge fields and multi class. This system has several parts (Xu, 2002), such as: conveyer device, the weight sorting device, the appearance sorting device, and the quality sorting device etc. (Fig. 2). 


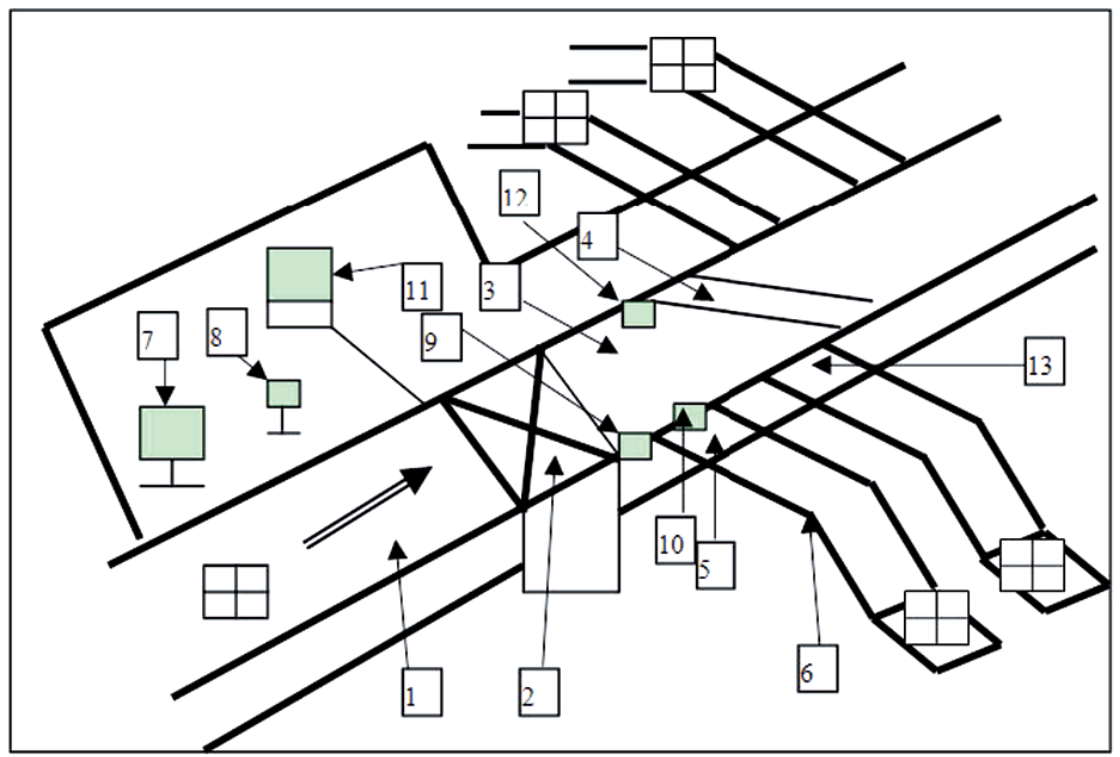

Fig. 1. The hardware diagram of fruit automated sorting system (1) input conveyer belt; (2) fill cargo conveyer belt; (3) steel conveyer belt; (4) Diverter; (5) output Rollers; (6) sorting port; (7) signal giver; (8) laser ISBN reader; (9) pass detector; (10) magnetism signal generator; (11) controller; (12) magnetism signal reader; (13) full scale detector.

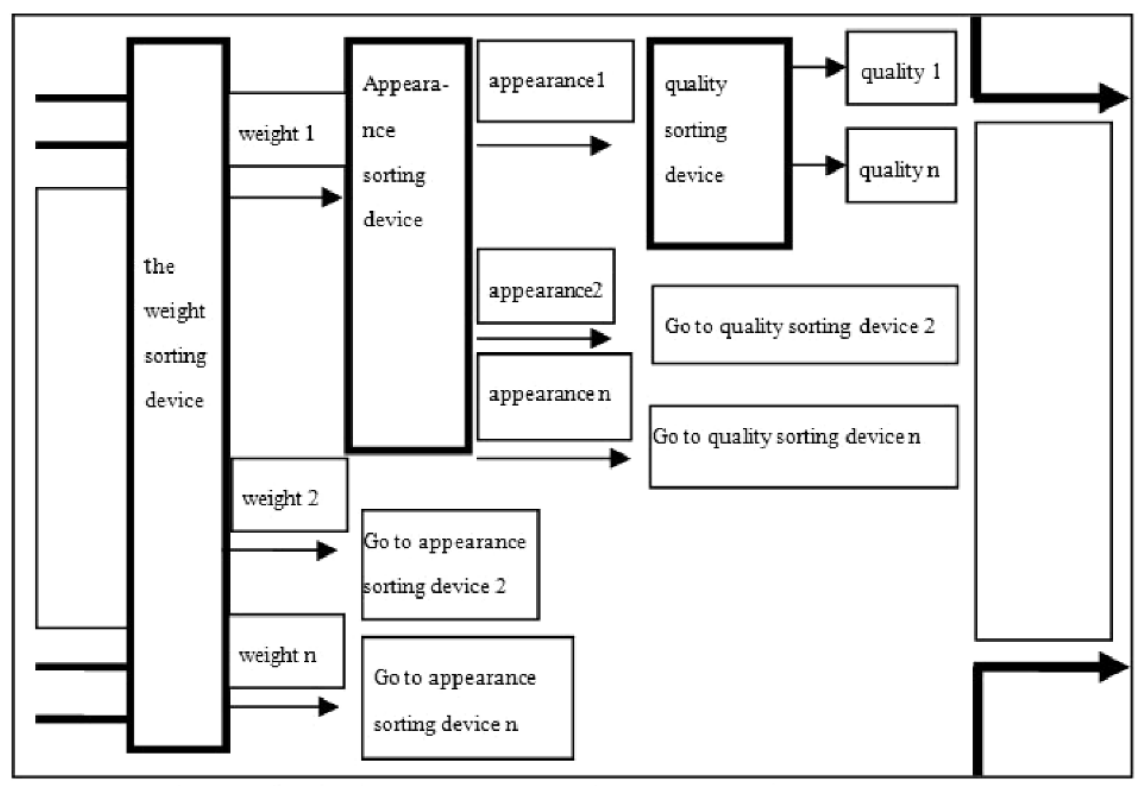

Fig. 2. The hardware consists of fruit automated sorting system 
The function module of the fruit automated sorting system is adapt to its The hardware consist, three module, which is weight sorting module, appearance sorting model and quality sorting module, is concluded in the system. The work principles of the each function module can be explained by the Figure (Fig. 3) (Han, 2004).

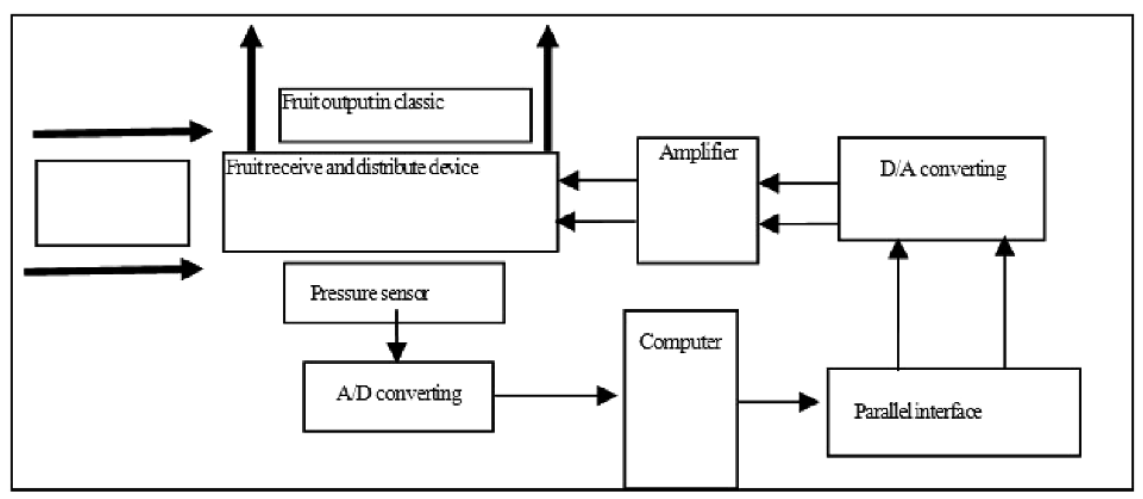

Fig. 3. The hardware consists of the weight sorting device

\subsubsection{The weight sorting device and its work principle}

The fruit which is need to be sorted is transferred to the fruit receive \& distribute device by conveyer belt (Fig. 3), the fruit receive $\&$ distribute device can detect the weight information of fruit by pressure sensor, the analog weight information from pressure sensor is transferred to digital information by A/D switch (Zhu, 2001), the digital information is sent to the computer, the computer process these information by special software, the result from computer is sent to different passageway by parallel interface (the purpose is to control the controller from fruit receive \& distribute device which can control the destination of the fruits), the digital information from parallel interface is convert to the analog signal by D/A switch, the analog signal is magnified by signal amplifier and it is sent to the fruit receive \& distribute device in order to control the Flow Direction of the different weight fruit. In order to enter the next sorting step, which is appearance sorting, the fruit from weight sorting device is sent to the appearance sorting device to be sorted by appearance sorting device. 


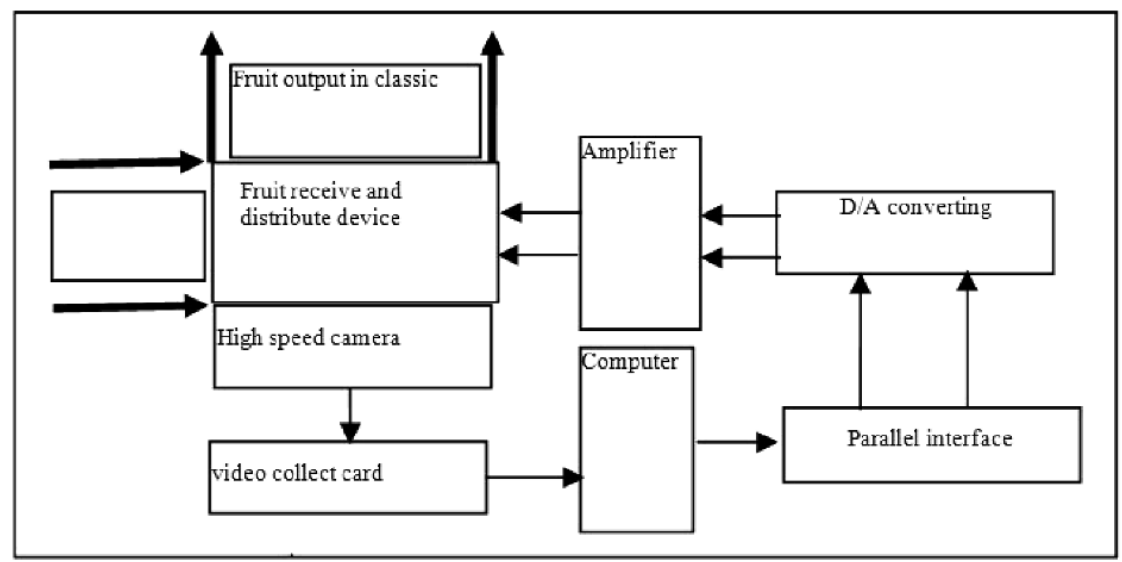

Fig. 4. The hardware consists of the weight sorting device

\subsubsection{The appearance sorting device and its work principle}

The fruit from weight sorting device is sent to the fruit receive \& distribute device of the appearance sorting device (Fig. 4), the high speed camera shoot fruit from various angles, the image from camera be sent to the computer by the video capture card, the computer process the video information by special software, the result from computer is the classified signal of fruit, the signal open the different sorting passageway by parallel interface, and then the signal is magnified by the signal amplifier. In order to control the fruit receive \& distribute device, the fruits are sent to the different passageway, and at last the fruit can be sent to the package device.

The image from camera should be processed by edge detection and noise removing (even it must be curve fitting) (Guo, 2004), and then it be matched to different fruit appearance so that the fruit can be sorted by appearance.

\subsubsection{The quality sorting device and its work principle}

The two devices, which is the quality sorting device and appearance sorting device, have the similar work process (Fig. 4). there are two different: one different is that the high speed camera must be infrared high speed camera; another different is that they have different process software .the software of the quality sorting device can process the fruit appearance quality (such as color and luster, stain etc) and inner quality (it is rot or not) according to the image from camera. It sorts manly according to the image from the infrared high speed camera and the trade standard. In the process of sorting, it makes full use of the digital image processing technology and Pattern Recognition technology. In general, the first step is to screen 
according to color and luster and stain, the second step is to screen the inner quality of the fruit according to the infrared image.

\section{THE SOFTWARE DESIGN OF FRUIT AUTOMATED SORTING SYSTEM}

In the course of the designing software of the System, different development tool should be thought because it refers to the hardware. the software of weight sorting and the computer interface is need to be developed by assembler programming language because it refers to hardware, other software, such as the digital image processing, the serial interface communication etc, can be developed by $\mathrm{Vc++}$, It has several special Control in Vc++ about serial interface communication (Dong, 2005).

\section{SUMMARY}

The fruit automated sorting system refers to different technology form different subject, such as automatic technology, the software and hardware technology of computer. The digital image processing technology, the artificial intelligence technology etc. the paper introduced the design thought of fruit automated sorting system, the consists of hardware, work principle and the design of software. The final application of the system can bring about large social benefit and economic benefit to agriculture production and management.

\section{REFERENCES}

Dong S. L. Application of PLC in the Automatic Sorting System, Machine Tool \& Hydraulics, 2005, 5:136-147.

Guo X. F., Liu W. T. An Automatic Feather Selecting System by Image Processing, Modern Electronic Technique, 2004, 3:30-33.

Han Y. M., Wang D., Wei H. H., Tan C. Q. The Design of the Parcel Automatic Sorting System, Mechanical \& Electrical Engineering Magazine, 2004, 8:11-12.

Huang Q. M. Automatic Sorting System and Analysis of application prospect, Logistics Technology, 2002, 5:7-15.

Xie L. X., Yang H. Y. Design and Implementation of Statistic and Analysis System for Automatic Luggage Sorting System, Computer Engineering (Supplementary Issue), 2005, 31:3-6.

$\mathrm{Xu}$ S. Y. Automatic Sorting System and the application, Logistics and Material Handling, 2002, 3:33-39.

Zhu Z. T. The design of computer auto-sorting system, Computer Engineering and Design, 2001, 6:66-88. 